\title{
APORTES DE LA RECREACIÓN EN LA APROPIACIÓN DEL ESPACIO PÚBLICO $^{1}$
}

\author{
Recebido em: $17 / 12 / 2016$ \\ Aceito em: 08/06/2017 \\ Astrid Bibiana Rodríguez Cortés ${ }^{2}$ \\ Universidad Pedagógica Nacional \\ Bogotá - Colômbia
}

RESUMEN: El presente artículo busca visibilizar los aportes que hacen la recreación en la construcción de un espacio público accesible, integrador y placentero, siendo este base estructural de una buena calidad de vida. El uso del espacio público con actividades recreodeportivas crea lazos físicos y simbólicos entre el espacio y las personas, además de fomentar el encuentro social, la participación ciudadana y el cuidado ambiental. Desde esta perspectiva se analiza la Ciclovía de la ciudad de Bogotá, un espacio público de recreación masivo que ha promovido la integración ciudadana y la convivencia social. Este espacio usa como mediación la actividad física y la recreación para incentivar la ciudadanía activa. Administrada por el Instituto Distrital de Recreación y Deporte, la Ciclovía, además de convertirse en baluarte de la capital colombiana, también ha sido ejemplo de "buenas prácticas", su modelo se ha replicado en diversos lugares de América. Pese a que se ha logrado señalar los beneficios de los programas masivos de recreación y deporte en la calidad de vida de los bogotanos, la inversión económica en este campo sigue siendo insuficiente. Por tanto, es primordial generar indicadores sociales que sustenten la necesidad de una mayor inversión así como evidenciar aún más los aportes de este campo en la construcción de espacios públicos colectivos que interpelan la propuesta de ciudades atomizadas y ciudadanos atemorizados e individualistas.

PALABRAS CLAVE: Actividades Recreativas. Recreación. Instalaciones Deportivas y Recreativas.

\section{RECREATION CONTRIBUTIONS IN PUBLIC AREA APPROPRIATION}

ABSTRACT: This document aims to show contributions that recreation performs over construction of approachable, integrator and pleasant public space, being structural base of good quality life. The public space use with recreational-sports activities creates physical

\footnotetext{
${ }^{1}$ Este artículo hace parte de la tesis de doctorado denominada La ciclovía de la ciudad de Bogotá: Dispositivo productor de subjetividades.

${ }^{2}$ Docente-Investigador de planta de la Universidad Pedagógica Nacional, Facultad de Educación Física. Doctor en Estudios Sociales de la Universidad Externado de Colombia. Magister en Educación.

Licere, Belo Horizonte, v.20, n.2, jun/2017 
and symbolic ties between space and people, as well as fostering social encounter, citizen participation and environmental care. Since this perspective the Bogota Ciclovía has analyzed, as public space of massive recreation that has promoted the citizen integration and the social coexistence. This space uses as mediation physical activity and recreation to encourage active citizenship. Managed by the District Institute of Recreation and Sports, Ciclovía, besides becoming a bulwark of the Colombian capital, has also been an example of "good practices", its model has been replicated in several places in America. Despite fact that benefits of mass recreation and sports programs to Bogotá citizen's quality life have been noted, economic investment in this field is still insufficient. Therefore, it is essential to generate social indicators that support the need for bigger investment as well as to highlight even more the contributions of this field in the construction of collective public spaces that challenge the proposal of atomized cities and frightened and individualistic citizens.

KEYWORDS: Recreation. Leisure Activities. Sports and Recreational Facilities.

\section{Introducción}

La concepción sobre espacio público en la sociedad colombiana es una aspecto relativamente reciente para Pardo (2008) fue en los años ochenta, cuando este concepto se instaló en las facultades de arquitectura y administración pública. El deterioro, la marginalidad, la desintegración y la violencia ciudadana, fueron detonadores de orden social que plantearon pensar que la recuperación de los espacios públicos constituiría una ciudad más vivible, integradora y equitativa.

Sin embargo, si bien se reconoció académicamente la necesidad de estudiar y generar proyectos para comprender y establecer la importancia social que tiene el espacio público en el acontecer ciudadano, la posibilidad de expresión política en estos espacios ha sido regulada y controlada a lo largo del siglo XX, tal como sucedió en Bogotá en la década de los noventa, por un lado, la ciudad se vio más cuidada y estéticamente mejor, y por otro, las manifestaciones ciudadanas en el espacio público se hicieron cada vez más restringidas y reducidas, entendiendo que éste es un aspecto fundamental para el ejercicio de los derechos políticos (ZAMBRANO, 2003). 
Uno de los propósitos fundamentales del espacio público para la ciudad es brindarse como espacio de esparcimiento y disfrute ciudadano. Si bien algunas miradas reduccionistas asumen al espacio público como un espacio físico de la ciudad, esto niega quizás una de sus concepciones más importantes y es precisamente ser elemento sustancial y articulador de las relaciones sociales ciudadanas. La posibilidad de encuentro y goce colectivo se produce en la medida en que el espacio público se disponga para ello. Una ciudad que permite que sus ciudadanos se encuentren plácidamente es una sociedad que tiende a proyectos cada vez más democráticos.

El incremento de parques, plazas y alamedas en la ciudad de Bogotá ha sido importante a lo largo del siglo XX. Estos fueron ganados gracias a la presión de la comunidad, sin embargo por sí solos estos espacios no se aprovechan en su máxima expresión, de allí que las prácticas deportivas y recreativas han permitido desarrollar en los usuarios sentimientos de aprecio, cuidado y conservación de los espacios públicos de la ciudad. En la medida en que estos son usados, ocupados y apropiados generalmente se convierten en espacios determinantes para el ejercicio de la ciudadanía.

Para comprender de manera amplia la problemática del espacio público y su importancia en la calidad de vida de los habitantes, se propone discernir en este documento sobre tres aspectos: primero, las concepciones que han existido sobre espacio público, segundo acercamiento a la configuración del espacio público en Bogotá y tercero señalar cómo las prácticas de recreación y deporte han contribuido en la apropiación y cuidado del espacio público bogotano. 


\title{
Aproximaciones a Concepciones sobre Espacio Público
}

La aproximación a la categoría de espacio público requiere una mirada multidimensional, polisémica y compleja puede

\begin{abstract}
Abarcar aspectos muy abstractos desde la filosofía y la teoría social, política y jurídica. En el otro extremo se sitúan trabajos muy concretos realizados por geógrafos, antropólogos, etnólogos, sociólogos, activistas políticos, psicólogos, periodistas, etcétera[...] y en otra dimensión se colocan las obras de urbanistas planificadores, arquitectos, cientistas sociales especializado en lo urbano que analizan el espacio público a partir de la relación entre las formas urbanas y los usos sociales. Y hay muchas otras perspectivas para tratar el espacio público (BORJA, 2014 p.14).
\end{abstract}

En tal sentido, no se pretende establecer una única concepción sobre este extenso campo, por el contrario, se quiere hacer un llamado para mirar con amplitud el tema, resaltando su dimensión de disfrute e integración colectiva. Un primer elemento a tener en cuenta es que el espacio público como lo diría Lefebvre (1974/2013) es una producción social $^{3}$ en un contexto histórico, por tanto, partir de una perspectiva lineal puede caer en el riesgo del anacronismo, sin embargo, si es posible establecer algunas condiciones sociales que han permitido darle sentido a ciertos usos y prácticas que han construido nociones sobre espacio público en momentos históricos determinados.

Para Zambrano (2003) el espacio público “[...] ha existido desde que se construyeron las primeras ciudades, pues la presencia de un centro urbano conllevaba la existencia de calles, plazas y otros lugares de uso común para los habitantes” (p.35).

\footnotetext{
${ }^{3}$ Lefebvre (1974) recurre a Marx y Engels para mostrar como los seres sociales, producen su vida, su historia su conciencia, su mundo. Por ello los seres humanos han producido formas políticas, jurídicas, religiosas, artísticas, filosóficas e ideológicas. Por tanto, para Lefebvre el espacio es una relación social, que se produce $\mathrm{y}$ que en consecuencia es un medio que produce a su vez unas relaciones, usos y formas productivas. 
Siguiendo al autor, el espacio público fue planteado en términos muy puntuales en el marco de la burguesía liberal, por tanto sus sustentos ideológicos tienen un fundamento en la revolución francesa: igualdad, libertad y fraternidad. Por tanto, el espacio público no puede verse como un simple espacio físico para transitar o desplazarse en las ciudades, su riqueza está precisamente en la dimensión política y social que dispone.

Sin embargo es innegable como la edificación de las ciudades ha en alguna medida minado la posibilidad de lo "público" que tiene el espacio, en tal sentido, Sennet (2011), muestra cómo la visión de lo íntimo induce a un abandono del dominio de lo público. La proyección y organización de las ciudades privilegian la construcción de espacios privados que precisamente protegen la intimidad con una de sus características principales, el "aislamiento". Los espacios sociales en la ciudad y las relaciones personales se hacen cada vez más íntimas a lo largo del siglo $\mathrm{XX}$, un hombre que transite rápidamente y $\sin$ comunicación alguna con el medio, es un modelo que se impone en las urbes, la velocidad, la transitividad y la celeridad son todas características de una vida moderna de eficiencia y producción. Desde esta perspectiva Sennet (2011) plantea el espacio público muerto "es una razón, la más concreta, para que las personas busquen en el terreno de lo íntimo lo que se le ha negado en un plano ajeno" (p.30).

Uno de los primeros usos del vocablo "público", estuvo asociado a la audiencia a las obras de teatro, años más tarde, se relaciona con las reuniones sociales de la burguesía y el interés por socializar en diferentes espacios, haciéndose este más cercano al significado actual. Por tanto, "público" establece una vida que transcurre fuera de la familia y amigos cercanos, llevándose a cabo en la ciudad, que se hace foco de la vida pública (SENNET, 2011). La necesidad de relación social en los espacios públicos genera la construcción de Licere, Belo Horizonte, v.20, n.2, jun/2017 
lugares y espacios que proponían unas formas de relación más tranquila, desprevenida y abierta entre los ciudadanos, pero también allí se impusieron patrones de clasificación social.

Es de resaltar cómo el surgimiento del espacio público tiene que ver con la necesidad que tiene la sociedad de recrearse y establecer otras formas de encuentro fuera de su círculo familiar. Puede decirse que algunos de estos espacios públicos se construyen como una manera de resistirse al control que se ejercía en la relación social regulada. Con el paso de los años las tensiones entre vida pública y privada se acentúan queriendo demarcarse con mayor claridad las fronteras entre lo uno y lo otro. Aún así, la proliferación de nuevos lugares, posibilitó otros modos de uso, generando a su vez otras prácticas espaciales en donde las demostraciones afectivas, la forma de vestir y el relajamiento social propusieron diferentes modos de intercambio social.

Finales del siglo XVIII surge en Inglaterra los parques públicos, los cafés, cafeterías y centros sociales. Así también espacios como teatros y la opera abrieron al público (SENNET, 2011). Este momento histórico permitió que la recreación ciudadana pululara con diferentes prácticas, permitiendo que la sociedad trabajadora organizará sus actividades de esparcimiento y socialización, disminuyendo la exclusividad que tenían las clases sociales más pudientes a estas actividades, tal como sucedía con los paseos por los jardines que eran de carácter privado y excluyente.

Las sociedades modernas, modificaron las relaciones sociales, los modos de uso y el intercambio comercial, alterando sustancialmente la ocupación del espacio público. Para Zambrano (2003) “[...] la consolidación del capitalismo en Europa y Estados Unidos creó unas nuevas relaciones sociales que exigieron mayores libertades individuales de Licere, Belo Horizonte, v.20, n.2, jun/2017 
circulación y mercado (p.36)". Esta concepción sobre las relaciones sociales condujo a replantear los espacios públicos y privados de la ciudad, una mayor sociabilidad implicaba disponer de espacios de libre acceso, mayor circulación y nuevas formas de consumo, pero a su vez otras maneras de controlar a la población, por ello, estos espacios también se jerarquizaron y ejercieron discriminaron por la raza, el género y la clase social, entre otros. Por otro lado, también se instituyeron como espacios de resistencia, asumiéndose en ellos prácticas de disfrute y otras maneras de comportarse socialmente.

Recientemente la connotación más usada y conocida es que el espacio público es un espacio opuesto a lo privado, en donde lo público es de todos o de interés común. Esta forma de definirlo llevó a que se legislara sobre él proponiendo un concepto jurídico:

Un espacio sometido a una regulación específica por parte de la administración pública, propietaria o que posee la facultad de dominio del suelo y que garantiza su accesibilidad a todos y fija las condiciones de su utilización y de instalación de actividades (BORJA, 2000, p.69).

Las sociedades modernas se dieron a la tarea de separar lo legal, es decir, la propiedad privada urbana expresada en el derecho a construir y la propiedad pública que supone un suelo libre de edificaciones y tiene como fin los usos sociales (esparcimiento, actos colectivos, actividades culturales, comerciales, etc.). Aún frente a estas divisiones y regulaciones, el espacio público tiene una dimensión determinante, la dimensión sociocultural, que establece una dinámica cultural creando espacios públicos que jurídicamente no fueron previstos como tal. Así, esa capacidad de interacción y actividad permite al espacio público hacerse cambiante y creativo, desbordando los conceptos y usos establecidos (BORJA, 2000). 
Hoy en día la dinámica social es amplia, gran parte de la vida de los ciudadanos se desarrolla en el espacio público, por ello se hace imprescindible el aporte de todos para mejorar la calidad de vida común. Es indiscutible la fuerza que tiene el espacio público para disponerse como espacio de intercambio social, expresión e integración ciudadana siendo estos argumentos suficientes para trabajar mancomunadamente en su diseño, transformación y cuidado. En tal sentido, se hace vital reconocer las múltiples identidades que históricamente se ha constituido en la ciudad, con el fin de determinar lógicas de organización propia, diseños que conserven la tradición cultural y posibiliten la invención de usos y prácticas espaciales que desplacen modelos funcionalistas del urbanismo.

En esta vía la propuesta de Carlos Mario Yory (2011) puede ser válida, este autor plantea la urgente necesidad de resemantizar lo que se ha comprendido como contrato social, para entenderlo más bien como acuerdo social ciudadano. Mientras el contrato se estable como una forma de control (frecuentemente establecido por quien detenta el poder) con relación al cumplimiento de unas determinadas "normas sociales" y por tanto supone unos resultados, el acuerdo social ciudadano supondría reconocer un proceso de autoorganización grupal por parte de los distintos actores interesados, así como unos intereses puntuales y unas formas de resolución propia, además el contrato social se establece previamente los interesados, las maneras y las temáticas a resolver, por tanto, el contrato "sirve para todo" en atención a la constitución y a la ley, en tanto, el acuerdo responde a situaciones y acciones concretas según cada caso.

Esta perspectiva supondría un compromiso ciudadano que permita establecer problemáticas que aquejan a la mayoría, reconociendo el beneficio común y no solo el bien particular, pensando así en establecer acuerdos más equitativos entre lo individual y lo Licere, Belo Horizonte, v.20, n.2, jun/2017 
colectivo, entre lo privado y lo público. Este ideal, "este deber ser" que aquí se propone, no puede dejar de lado la realidad que acoge la compleja estructuración de la ciudad hoy, los diversos acontecimientos sociales acaecidos en la modernidad son marco de análisis de los fenómenos híbridos y complejos que acompañan la emergencia de lo urbano.

Es indiscutible la influencia que ha tenido el urbanismo en la estructuración de formas de cultura urbana que la distingue de lo rural, concebido al principio del siglo XX lo primero como refinado y culto y lo segundo como poco desarrollado e ignorante. Por ejemplo, en la ciudad de Bogotá desde mediados del siglo XX, con la idea del desarrollo producto de la dinámica social después de la Segunda Guerra Mundial. Los países occidentales intentaban construir un orden mundial unificado encontrándose a la cabeza los Estados Unidos quien impuso una idea de desatrasar a los países del tercer mundo ${ }^{4}$, sellado esto con un pacto desequilibrado y peligroso como fue la “Alianza para el progreso". Así, el desarrollo se erigió sobre la base de condiciones de productividad de los países del primer mundo, de tal manera que los patrones de riqueza de un grupo de naciones occidentales han constituido los parámetros sobre los cuales se miden los niveles de desarrollo de los países del Tercer Mundo. Las condiciones de vida de esas naciones privilegiadas, se utilizan como medida para saber si pertenece al primer o tercer mundo.

De esta forma se opto por un modelo de ciudad desarrollista, quien con la importación de un modelo de ciudad funcional centrado en una idea de modelo espacial de grandes avenidas y edificios y estéticas asépticas desplazaba:

\footnotetext{
${ }^{4}$ Cfr: Arturo Escobar (1996). La invención del tercer mundo: construcción y deconstrucción del desarrollo. El análisis de este autor permitirá establecer cómo nunca ha existido un tercer mundo, ha sido una estrategia para someter a las naciones menos adineradas a seguir modelos políticos, económicos y sociales establecidos por los países potencia. Estableciendo un orden global que los favoreciera.
} 
La parte árabe del modelo hispanomusulmán de ciudad que hereda Latinoamérica; hispano, en lo concerniente al uso administrativo (racional, eficiente, concéntrico, jerárquico y funcional) que este hace de la malla urbana (razones por las cuales sobrevive en el nuevo modelo); y musulmán, en lo que se refiere al uso de la calle como espacio de vida, encuentro e intercambio, habida cuenta del imaginario del bazaar que alienta el mercado informal (mercado persa) y, con él de la "indeseable" presencia (para el urbanismo anglosajón que imitamos) de los "milenarios vendedores ambulantes (razón por la cual no sobrevive, o lo hacen a expensas de la estigmatizante figura de la marginalidad que transforma la informalidad en ilegalidad) (YORY, 2011, p. 119).

Aún ante los Planes de Ordenamiento Urbano concebidos para organizar a Bogotá desde los años treinta del siglo $\mathrm{XX}$, $\mathrm{y}$ con algunos avances en términos de edificación $\mathrm{y}$ organización vial, los ciudadanos no acatan del todo sus lineamientos. Así, los modelos que establecían una ciudad estéticamente bella y funcionalmente de avanzada, han tenidos algunos detractores, entre otros: los orígenes establecidos como los comenta Yory, la fuerte migración rural que sufre constantemente la ciudad, los fenómenos sociales de desigual, violencia, además de unas administraciones poco claras y con deficiente planeación, factores todos que no han permitido la implementación en su totalidad de modelos importados de ciudad.

La tensión entre la imposición de modelos de un urbanismo funcionalista con la idea de edificar estereotipos de ciudad avanzada que no corresponden a la realidad social y la defensa que han consolidado los movimientos sociales desde mediados de los años setenta de su derecho a la expresión ciudadana, la preservación de espacios y la estructuración de otras formas de organizar la ciudad, han traído la implementación de formas de territorialización del espacio público. Tensión que no solo se presenta en la ciudad de Bogotá sino en general en las ciudades Latinoamericanas. 
Pese a la tensión establecida entre la imposición del Estado por modelos urbanísticos y la resistencia social, la ciudad, en el caso de Bogotá ha venido lentamente avanzando en establecer acuerdos ciudadanos colectivos que propenden por encontrar estilos propios de organización y regulación social que retoman el valor simbólico, social y expresivo que tiene lo popular en la construcción de lo público en el espacio. Como ejemplo se encuentra la revalorización del centro histórico de la ciudad, el cual ha incorporado un estilo de conservación y preservación conjugado con nuevos elementos de cultura popular. Además se destaca en estas prácticas de conservación del patrimonio arquitectónico, la participación y concertación que se ha tenido con los gobiernos locales y los gestores culturales. En alguna medida y si bien este proceso no ha sido sencillo, ni se cumple a cabalidad, se han dado pasos para pensar una política que permita al ciudadano, el usuario común tomar conciencia de su responsabilidad y los derechos que debe asumir en acciones puntuales como el uso, cuidado, preservación, conservación, entre otros de los espacios físicos, así como, al Estado para comprender la necesidad de abrir su perspectiva al acuerdo y la participación de los diferentes actores que implica la gestión del espacio público, que conlleva a desafíos más importantes que el tema de la preservación del patrimonio histórico, sin que ello no sea importante, y es precisamente ejercer una dinámica social entendida como la legitimación de un conjunto de derechos políticos, sociales y cívicos que redunden en la convocatoria pública, la expresión colectiva y la integración social. En este sentido de acuerdo con Borja:

El espacio público nos interesa principalmente por dos razones. En primer lugar, porque es donde se manifiesta muchas veces con más fuerza la crisis de "ciudad" o de "urbanidad". Por lo tanto, parece que sea el punto sensible para actuar si se pretende impulsar políticas de "hacer ciudad en la ciudad". Y en segundo lugar, porque las nuevas realidades urbanas 
especialmente las que se dan en los márgenes de la ciudad existente plantean unos retos novedosos al espacio público: la movilidad individual generalizada, la multiplicación y explicación de las "nuevas centralidades" y la fuerza de las distancias que parecen imponerse a los intentos de dar continuidad formal y simbólica a los espacios públicos (BORJA, 2000, p.68).

El espacio público se enfrenta día tras día a problemáticas más complejas y difíciles de atender, si se promueve aquí como se ha visto la vía de los acuerdos, es importante determinar acciones concretas que puedan ser identificadas por los ciudadanos como caminos para continuar. Lo público requiere entonces ejercer su dominio, su apropiación y darle un sentido. Se supone incentivar el uso de los espacios, el cual se caracteriza por la accesibilidad que pueda tener la población al espacio, así también se encuentra la calidad del espacio que puede ser medido como lo propone Borja "por la calidad de las relaciones que facilita, por su fuerza mixturante de grupos y comportamientos y por su capacidad de estimular la identificación simbólica, la expresión y la integración cultural” (pág. 68). En tanto, es fundamental trabajar desde los diferentes sectores y actores por un espacio público de calidad para todos, es decir, accesible contar con los espacios y equipamientos para ser disfrutados, dinámico, que encuentre respaldo financiero para ser promovido con programas y proyectos que permitan la inclusión e integración social y permanente establecer políticas para mantener el dinamismo, el cuidado y la apropiación ciudadana.

Se puede decir entonces que se entiende por espacio público como eje estructural de una ciudad incluyente, accesible y equitativa que permite el encuentro social y cultural, así como la posibilidad de construir en conjunto formas alternativas de vivir, apropiarse y empoderarse de la ciudad, en otras palabras, de ejercer una ciudadanía activa. En tal sentido, en el próximo apartado se quiere destacar cómo las prácticas recreativas han Licere, Belo Horizonte, v.20, n.2, jun/2017 
aportado en la apropiación de espacio público, generando formas para que los ciudadanos se relacionen de una manera distinta con su ciudad. Se analizará el caso de la ciclovía de la ciudad de Bogotá en sus cuarenta años de desarrollo, así como las tensiones y dificultades que se han establecido en este proceso.

\section{Aportes de la Recreación al Espacio Público Bogotano}

Bogotá es la capital de Colombia, es la ciudad más grande y poblada por personas de distintos lugares del país. Lo que hace que el aprecio que se sienta por esta ciudad no sea un acto natural, es decir porque muchos de sus habitantes no nacieron allí, en algún sentido, hablar de apropiación del espacio tiene que ver con la filiación que se sienta con ellos, ya sea por haber nacido en este territorio, por tradición, o por agradecimiento con el lugar donde se habita. En el caso de esta urbe, los estudios han mostrado que existe un gran desarraigo la ciudad es vista con un fin más utilitarista que una ciudad para disfrutar y vivir plácidamente. Para Armando Silva en los estudios realizados sobre imaginarios urbanos, muestra

Como existe una relación entre lo feo e inseguro de Bogotá, y el carácter agresivo que se le adjudican a sus ciudadanos, con el nulo sentido cívico ciudadano. Todas estas circunstancias apuntan hacia una limitada calidad de vida, a pesar de la infraestructura, servicios, redes de salud y parques recreativos Bogotá ocupa un relativo lugar intermedio entre las grandes ciudades del mundo, y a pesar de significativos alcances en prestación de servicios urbanos (SILVA, 2006, p. 208).

La percepción que tiene el habitante sobre su ciudad, lugar donde habita y transcurre su cotidianidad, construye formas de relación con los espacios, por esto abordar el tema del apropiación del espacio público tiene que ver con reconocer los aportes que 
distintas disciplinas han hecho para explicar los modos, prácticas y formas de usos, de vivir y empoderarse de un espacio que es de todos.

Desde los presupuestos de geografía humana Alicia Lindón (2014) se ha venido preguntando por el habitar la ciudad, entendido por ello, como una experiencia, el hacer, las prácticas, el movimiento constante de la vida cotidiana de todo sujeto, centrando su interés en los lugares urbanos. Para Lindón es importante comprender los sentidos que puede tener un lugar para el sujeto que lo habita, pues este puede darse del

Entrecruzamiento de la percepción de las materialidades del lugar, de la propia performatividad del sujeto-cuerpo que practica el lugar, de los vericuetos de la memoración de lugar que realiza cada sujeto, de las emociones que cada lugar activa en un sujeto y las tramas de sentido que los sujetos hacen y rehacen permanentemente en relación con sus lugares habitados (2014, p.61).

Todo lo anterior muestra la complejidad al analizar lo que implica habitar un lugar, este fenómeno hace parte de la biografía de los habitantes, por ello, los estudios de Lindón se acercan a establecer esta relación del sujeto con el espacio de manera profunda, además se aproxima realizando una compleja red topológica que integra los lugares habitados por el sujeto. Su aporte para el caso que compete a este documento en términos de apropiación de espacio, está dado en la medida que muestra la filiación que ejercen los sujetos con los espacios, independiente de la expresión pública que pueda hacer de ello. Para Lindón debe superarse la vieja dicotomía entre los espacios públicos y privados, proponiendo estudiar cómo la red topológica de habitares integra los dos aspectos (público-privado) que articula visibilidades e invisibilidades, cierres y aperturas. Esta perspectiva de estudio implica reconocer las experiencias de los sujetos en los espacios, su red topológica (los lugres por donde frecuentemente transita) y el entrecruzamiento que ellos tienen en su biografía, Licere, Belo Horizonte, v.20, n.2, jun/2017 
quizás esta perspectiva permita dar cuenta de la escasa conciencia que tienen los habitantes del espacio donde generalmente vive y lo mucho que puede afectar sus cambios en el desarrollo de la vida.

Una de las disciplinas que ha aportado al tema de apropiación del espacio público es la psicología ambiental, sus contribuciones se centran en la relación entre entorno físico y psicología, los investigadores de esta línea revisan con atención la manera en que un espacio natural se transforma en un espacio construido que termina siendo significativo para las personas. El espacio deber ser entendido "[...] como un espacio sociofísico, cruce de características físicas, percepciones psicológicas y de significados sociales y culturales que configuran esos patrones con los cuales nosotros dialogamos, con los cuales interaccionamos" (VALERA, 2014, p. 99).

Los estudios en psicología ambiental han venido mostrando la importancia que tiene el espacio para las personas, donde establece cómo la relación que se produce en los espacios públicos es de carácter intersubjetivo, donde el individuo se reconoce en la medida en que los otros también lo reconocen en un espacio conjunto. Esta relación de sociabilidad y encuentro genera en el individuo apegos y bienestar emocional, transformando el espacio de un interés funcional a un interés simbólico que se incorpora a la vida cotidiana (VALERA, 2014).

Estas afirmaciones dan pie para considerar que la potencia del espacio público no está centrada en entenderlo como espacio físico, lo vital esta en los niveles de relación social que permite. En la cotidianidad los sujetos tienen unas redes topológicas como lo propone Lindón, que cobran sentido por las experiencias que se construyen en los lugares y el impacto que tiene en su acontecer cotidiano. 
Por tanto, la interacción social, la manifestación de ideas y las diversas formas de usos, son acciones que se desarrollan en el espacio urbano donde se ejerce la participación social, cultural y política, en tanto derecho como deber ciudadano. Hoy el espacio público se ve amenazado, por un lado, las políticas de densificación no lo protegen sino que lo vulneran, por otro lado, el miedo y la inseguridad de las grandes ciudades hace que las personas huyan de ella. En tal sentido, diversos investigadores (SILVA, 2006; PÉRGOLIS, 1998; PARDO, 2008) desde hace algunas décadas han llamado la atención del cuidado y la importancia de generar políticas y acciones rápidas, eficaces y eficientes para defender, recuperar, revitalizar, cuidar y mantener el espacio público como estructura de la vida social en la ciudad.

En este sentido, las prácticas de recreación y deporte vienen desde hace algunos años, haciendo un aporte importante al espacio público de la ciudad, la implementación de diversas actividades permite que los bogotanos se integren y participen en el uso, cuidado y mantenimiento de sus espacios, mejorando la calidad de vida de los habitantes. La Ciclovía es uno de los programas que se ha reconocido por su contribución en la apropiación de espacio público. Este programa es un evento “[...] cultural, recreativo y público de gran significación urbana, y representa uno de los escenarios públicos para el encuentro ciudadano más seguro y equitativo" (PARDO, 2008, p. 230).

\section{La Ciclovía de la Ciudad de Bogotá: Una Experiencia Urbana de Apropiación}

La historia de la ciclovía de la ciudad de Bogotá nace de una gran manifestación: "[...] para que todo el mundo monte en bicicleta", esa era la consigna que invitaba al 
circuito de la tranquilidad, "[...] no se hará a beneficio de ninguna obra social, ni para participar en él se necesita inscripción, tampoco se dará largada ni abra trofeos, nadie ganará ni tampoco habrá coleros." (CIRCUITO CICLISTICO DE LA TRANQUILIDAD, EL TIEMPO, 1974).

Este circuito organizado por Pro-Bicicleta ${ }^{5}$, se encargó de motivar a los bogotanos a participar con el ánimo de organizar la primera manifestación del pedal, su propuesta era visibilizar la necesidad de ciclorutas para la ciudad. La convocatoria era abierta, desde las 9 de la mañana hasta las 12 del medio-día, las carreras $7^{\mathrm{a}}$ y 13 entre las calles 34 y 72 , estarían cerradas para los automotores. Los ciclistas tendrían la posibilidad de entrar por cualquier calle y pasear. La vía estaría cerrada y para ello se contaría con el apoyo del Departamento de Tránsito y Transporte. Para Pro-bicicleta, esta manifestación, “[...] demostraría que la bicicleta es el mejor vehículo: no contamina el aire, no hace ruido, mantiene el estado físico y además es 'divertidísima'" (CIRCUITO CICLISTICO DE LA TRANQUILIDAD, EL TIEMPO, 1974).

El domingo 15 de Diciembre del año 1974, fueron cerradas por primera vez, algunas calles principales de la ciudad de Bogotá por el Departamento de Tránsito y Transporte, el cierre se dio con el fin de que algunos ciudadanos usuarios de bicicletas, se manifestaran. Esta acción fue denominada por el periódico El Tiempo como el Mitin a Favor de la Cicla. Se registró una asistencia de más de 5.000 ciudadanos, los cuales salieron a las calles para

\footnotetext{
5 El Periódico El Tiempo la enuncia como Pro-Bicicleta, más adelante la denominará Pro-Cicla, esta organización estaba conformada: Jaime Ortíz Mariño, Fernando Caro Restrepo, and Rodrigo Castaño Valencia. Estos tres jóvenes entusiastas de la bicicleta de clase media alta de la ciudad de Bogotá, usando las conexiones políticas de sus familias lograron los permisos respectivos para hacer la gran manifestación del pedal (MONTERO, 2016).
} 
protestar contra la contaminación ambiental, sus consignas eran ¡abajo la contaminación!, ¡viva el aire puro!,. Esta protesta se provocó por la proliferación de automóviles, y la marginación de la bicicleta en las principales vías de la ciudad.

Según el diario El Tiempo, a esta singular protesta asistieron decenas de ancianos, amas de casa, gerentes, funcionarios, niños, hippies y empleadas domésticas. Algunos de ellos pegaron carteles a sus bicicletas y pidieron "libertad" al gobierno. En la manifestación, varios de los asistentes se dedicaron a correr y otros aprovecharon la ocasión para montar a su familia en barra y portapaquetes (PALACIOS, EL TIEMPO, 1974). Del mismo modo, Procicla $^{6}$ planteó que esta organización llevaba ya algún tiempo exigiendo a los urbanistas de Bogotá “[...] planear la construcción de ciclovías alternas a las principales avenidas, como ya se ha hecho en muchas ciudades europeas y norteamericanas" (PALACIOS, EL TIEMPO, 1974).

En entrevista realizada por el periódico El Tiempo, a Jaime Ortíz Mariño ${ }^{7}$, uno de los organizadores de la protesta, comentó sobre la seriedad de esta manifestación y exigió seguridad y respeto para los ciclistas, al igual que los conductores de automóviles, aviones y motocicletas. Para Ortíz, se trataba de "[...] una masiva actitud humana relacionada con el medio en el que se desenvuelve las consecuencia de deterioro sistemático que va sufriendo la ciudad" (PALACIOS, EL TIEMPO, 1974).

La acción social del Mitin a favor de la cicla, permitió entretejer la fuerza de los movimientos sociales ecologistas y los aficionados al ciclismo recreativo o deportivo, con

\footnotetext{
${ }^{6}$ En el primer artículo el diario El Tiempo, nombra a la organización social que convoca Pro-bicicleta, en el segundo artículo la llama Procicla.

7 “Arquitecto de la Universidad Case Western Reserve University de Cleveland, Ohio (1966-1970). Profesor de Arquitectura de la Pontificia Universidad Javeriana. Fundador de Pro-Cicla, uno de los dueños del almacenes Ciclopedia, famoso almacén de venta de bicicletas en la ciudad de Bogotá" (MONTERO, 2016, p. 8). 
el objetivo de demandar una mejor movilidad y preservación de los recursos ambientales, a partir de una estrategia lúdica y divertida como sería un circuito en bicicleta por algunas calles de la ciudad de Bogotá.

Este acontecimiento se considera el primer antecedente histórico de la Ciclovía. Para Pro-bicicleta, lo importante era generar vías alternas para el tránsito permanente de las bicicletas, ganando para ellas un estatus como vehículo, tal como lo tenía el transporte público, las motos y los carros. Hoy lo que se conoce como Ciclovía es diferente, tiene una connotación social, prácticas, discursos e intencionalidades distintas, a los motivados por esa primera marcha.

En 1982 el Alcalde Augusto Ramírez Ocampo, será conocido como el Alcalde de la Ciclovía. Su exaltación por el uso del espacio público, fue una de las consideraciones importantes para promoverla como forma de reivindicación social. "Será su correcta administración, construcción, conservación y cuidado y su amplia e imaginativa utilización la que logre mejorar la calidad de vida de los habitantes”, expresará Ramírez Ocampo, en la presentación del libro Ciclovías Bogotá para el ciudadano de 1983.

En este texto, el Alcalde Ramírez Ocampo señaló la falta de cuidado y uso inapropiado de la infraestructura de la ciudad. Resaltó el proceso de desarraigo, falta de amor, orgullo e identidad, quizás por el desmedido crecimiento, el desorden en el perímetro urbano, el advenimiento desproporcionado de personas de todo el país, el abandono de los sitios históricos y la pérdida de lugares de encuentro. Por ello, las Ciclovías de Bogotá es una solución urbana que reivindica el espacio público y recupera para la ciudad su lugar de “[...] encuentro y a escala de la Bogotá actual dentro del más sano y alegre concepto de recreación en una atmósfera contagiosa de seguridad” (RAMÍREZ, 1983, s.p.). 
Fueron $56 \mathrm{Km}$ los que se acondicionaron, una red que intentó unir circuitos que estaban aislados en diferentes puntos de la ciudad. Para Ramírez Ocampo, "Este fenómeno social, es tan popularmente positivo y aceptable como gubernamentalmente irreversible". Por esta razón, el lema con el que se abrió el programa era Ciclovía para el ciudadano:

Es claro que el fenómeno deberá estudiarse, complementarse y desarrollarse. En primer término, se deberá ampliar la cobertura actual de las Ciclovías de Bogotá en sus áreas, circuitos y horarios de forma tal que se intercomuniquen todas, se interrelacionen sus parques y se les dé a los ciudadanos por medio de ellas acceso a los importantes eventos comunitarios hasta convertirlas en el principal medio de comunicación dominical de la ciudad (RAMÍREZ, 1983, s.p.).

Este Alcalde de forma explícita planteó el desarrollo y el aporte que sería la Ciclovía para Bogotá, propuso de manera abierta, la importancia que a futuro podría tener la bicicleta para la movilidad. Estableciendo cómo en los años venideros los problemas de contaminación ambiental, tratamiento adecuado del espacio público, cantidad exacerbada de automóviles, encarecimiento del combustible y del servicio, la dificultad en los desplazamientos, serían algunos de los aspectos que sugería a la Ciclovía como una alternativa nueva e inmediata para los problemas de movilidad. La bicicleta era un vehículo de bajo costo que fomentaba el comportamiento ecológico, con una muy buena aceptación popular y con excelentes resultados en países avanzados. Son tres los aspectos que resalta Ramírez Ocampo en este programa: lo ambiental, la construcción de ciudadanía y lo recreativo como agente socializador.

Es de subraya el cambio que existe en la concepción de espacio público en las administraciones de Bogotá de los años setenta a los ochenta. En los setenta la administración de la ciudad se centró en la modernización y en la responsabilidad que el 
Estado tenía en velar por él. Comienzos de los ochenta, la concepción era de bien inalienable, donde el ciudadano era importante para lograr su cuidado, reivindicación y bien común.

Se considero que la implementación de la Ciclovía fue un proyecto de inversión mínima y con un recaudo en términos sociales muy alto. Este programa se estableció como pilotaje alternativo por la Alcaldía de Bogotá. Los medios de comunicación registraron la llegada de la Ciclovía como el programa dominical de los bogotanos donde las personas en sillas de ruedas, jóvenes en patines, familias caminando, muestras de diferentes clases de prácticas deportivas, se encontraban en los kilómetros habilitados para tal fin. La Ciclovía se reconocía como espectáculo para la ciudad:

Ir a la Ciclovía como mero espectador es también asistir al mejor espectáculo que, sin actores, cuenta la historia del deportista profesional que madruga a las 7 de la mañana a entrenar, del aficionado ocasional que consagra dos horas a partir de las 8 de la mañana para practicar su deporte favorito y del ciudadano común que asiste a la Ciclovía después de las 10 para buscar amigos, para pasear a su familia, o sencillamente para "darse un baño de popularidad". En fin la Ciclovía es Bogotá en plan de descanso, ocio y alegría como nunca antes se ha visto (GÓMEZ, 1984).

La Ciclovía retoma la calle como protagonista de lo público, como aquello que por un lado pertenece a todos, pero también para la audiencia que presencia un evento. Para Saldarriaga (1999):

La diferencia semántica en el significado del término "público" parece tener una relación directa con la manera en que se mira y se maneja el espacio público en la ciudad. Para unos es el inmenso terreno donde la ciudad se reconoce a sí misma. Para otros, es un lugar hecho para ser observado a distancia, como si fuera un evento (s.p). 
Finalizando el gobierno del Alcalde Augusto Ramírez Ocampo (1983), deja la Ciclovía con $54 \mathrm{~km}$ de circuitos conectados, con la edición de un libro Ciclovías para los Bogotanos, con el Primer Seminario de Planeación y Diseño de Ciclovías en la ciudad de Bogotá. Entre los días 21 y 22 de octubre de 1983 y un deseo manifiesto que el programa se consolidará.

Finalizando la década de los ochenta el programa se mantuvo pero no con el mismo empuje de años anteriores, se determinó acortar sus kilómetros y fue poca la atención que se prestó. Sin embargo, se reconoció, tal como lo menciona Ruby Marcela Pérez, en su artículo Ciclo-solución a la Recreación, cómo el programa Ciclovía es un espacio público ganado por los ciudadanos para su recreación y esparcimiento:

Hay alienados que persisten en su mal. Es la deducción a la que llegaron los ambientalistas que, a comienzos de los años 70, propusieron que la gente se volcara a las calles por lo menos un día a la semana. Su discurso no fue bien aceptado. La recreación no se concebía sobre el pavimento. Pero la pelea por la obtención de espacio público todo terreno terminó ganándola la calle. Y ese invento, el de la Ciclovía, partió en dos la historia del tiempo libre en Bogotá. De ahí que no haya perdido vigencia en los casi veinte años de existencia (PÉREZ, 1992).

Finales de 1995 bajo la administración del Alcalde Antanas Mockus, mediante la firma de un Convenio entre la Secretaria de Tránsito y Transporte (STT) y el Instituto Distrital de Recreación y deporte (IDRD), se endilga la administración y control de la Ciclovía se al (IDRD), quien decide hacer un análisis del programa, teniendo en cuenta, el tipo de población y los tramos existentes en los 24 kilómetros entregados. Como resultado de este estudio se propuso diseñar circuitos en la ciudad haciendo una conexión con los parques, los escenarios deportivos y con los sitios turísticos, arquitectónicos, culturales e 
históricos de Bogotá (INSTITUTO DISTRITAL DE RECREACIÓN Y DEPORTE IDRD, 2007, p. 124).

Bajo la dirección del IDRD se encontraba Guillermo Peñalosa, quien se encargará de promover un cambio en la concepción, dirección y operación de la entidad, y en la Ciclovía como programa recreativo. Por lo tanto, el circuito se amplía a 84 kilómetros, organiza las ventas ambulantes y crea nuevos servicios y actividades como la Recreovía (MONTEZUMA, 2011, p.63). El objetivo principal del programa Ciclovía será, Formar

\section{Ciudad.}

En esta década el sentido de la recreación se modifica sustancialmente, las acciones del Alcalde Mockus, la proponen como mediadora, para lograr objetivos sociales. El movimiento de la Ciclovía y su transformación se empezó a notar, las personas respondieron al llamado del alcalde y del IDRD, la Ciclovía se recuperaba de una década de silencio y poca atención. Bajo la administración de Guillermo Peñalosa, la recreación, demostró su aporte en la construcción de ciudad con una inversión alta en el mejoramiento de los parques, la creación de nuevas escuelas de formación deportivas para niños y la incorporación de un programa recreativo para adulto mayor, el IDRD empezó a ser visto como un instituto importante para la ciudad, al cual, le había sido cuestionada su gestión en años anteriores.

En 1996, llegó a coordinar el programa de Ciclovía y Recreovía Martha Lucy Barriga, quien previamente trabajaba en la división de Recreación del IDRD, su forma de concebir la recreación se implementó en la Ciclovía que empezó a transformarla, en entrevista para el periódico El Tiempo en 1999 comenta: 
Actualmente, la Ciclovía es todo un complejo de diversión para los bogotanos, donde trabajan 2.500 personas cada jornada, entre guardianes, alfabetizadores, auxiliares bachilleres, instructores de aeróbicos (distribuidos en ocho puntos de la ciudad), monitores y agentes de tránsito. [...] Las Ciclovías son un espacio para la familia, donde pueden interactuar con las demás personas en un ambiente completamente seguro (NULLVALUE, 1999).

Con un grupo institucional al frente del programa y con una intencionalidad de la recreación como mediación de formación de la ciudadanía, la Ciclovía se volvía un gran experimento social. Los ciudadanos respondieron a los cambios, la asistencia era masiva y el programa creció exponencialmente, requiriendo que el grupo humano aumentara y se extendiera por la ciudad para mejorar el servicio, intentando que los ciudadanos se comportaran adecuadamente en este espacio público que era propuesto para el disfrute de todos.

Comienzos del siglo XXI la Ciclovía se convierte en un espacio público recreativo que se caracterizó por relaciones como: salud-actividad física, belleza-estética corporal, ecología humana-ambiente y entrenamiento físico-formación del carácter. Estas relaciones basadas en discursos científicos, conformaran una red que justificará y fortalecerá su permanencia. Sumado a la re-conceptualización del espacio público, como condición de calidad vida, disfrute y apropiación ciudadana, serán condiciones de posibilidad que harán del programa un patrimonio de la ciudad.

La llegada de Enrique Peñalosa a la Alcaldía (1998-2000) da un aire de seguridad y tranquilidad a la Ciclovía como programa en el IDRD. Desde su plan de gobierno, Peñalosa, se comprometió a fortalecerlo e impulsar sus acciones. En el año 2000 se separan administrativamente los programas de Recreovía y Ciclovía con el fin de optimizar sus 
servicios. Dos aspectos se destacaran en esta última década, por un lado, la relación saludejercicio que se desarrolla como horizonte de sentido del programa y por otro, el reconocimiento a la ciclovía como fundadora de una forma alternativa de movilidad en la ciudad.

En el año 2003, se realiza el estudio Línea base y evaluación de satisfacción del programa, a cargo de la Universidad Nacional de Colombia, el objetivo era evaluar el nivel de satisfacción de la población con los programas del IDRD. Para ello se realizan 76.200 entrevistas, de las cuales 35.700 fueron realizadas a los usuarios de Ciclovía. Recibiendo una nota de 81,1 . El 70\% de los usuarios a la Ciclovía son hombres, contra un 30\% de mujeres. El rango de edad de mayor asistencia es entre $19-35$ años con un 50\%, y siendo los estratos medios quienes más la frecuentan. El 33\% de los usuarios salen a Ciclovía por mantenimiento físico, el $28 \%$ por diversión y el $26 \%$ por salud. En este momento el $78 \%$ de los usuarios manifiestan estar satisfecho con el programa Ciclovía.

En el año 2009, en un segundo estudio de satisfacción, de manera más específica, sobre los programas de Ciclovía-Recreovía. Este estudio insiste cómo el espacio público es fundamental en la calidad de vida de los bogotanos:

Desde el punto de vista social éste debe ser un tema relevante para conseguir que los espacios públicos de la ciudad sean lugares desde donde se pueda mejorar la calidad de vida de sus habitantes mediante la satisfacción de las necesidades básicas que van más allá del simple alojamiento, pues habitar la ciudad implica también jugarla y disfrutarla. El espacio público constituye un referente para la construcción de identidad de las sociedades urbanas ya que permite tanto la re-creación de la historia común como la identificación de las personas con los lugares físicos (p. 5). 
Por tanto, la Ciclovía fortalece el sentido de apropiación del espacio público de la ciudad, favoreciendo la cohesión social y fomentando la participación comercial y económica de sus habitantes además de beneficiar la autoestima urbana. Según el informe, la Ciclovía responde a las demandas de participación, recreación y deporte de los bogotanos, generando mecanismos para transformar su entorno social y cultural. Su infraestructura posibilita la participación de más de (1.000.000) de usuarios, para la realización de actividad física. El informe también muestra cómo la Ciclovía se hace merecedora del reconocimiento del II concurso de ciudades activas.

En general el estudio realizado por la Universidad Nacional, destaca los beneficios de este espacio público con relación a la apropiación ciudadana, reconoce en este programa la posibilidad de acercamiento social y convivencia pacífica. Sobre la administración del espacio se enuncia como deficiente, muchos de los usuarios desconocen por completo que existe una entidad que regula y administra la Recreación y el Deporte de la ciudad. Una de las recomendaciones del estudio es fortalecer la comunicación institucional.

Uno de los aspectos que se destaca finalizando la primera década del 2000, es la Ciclovía, es un ejemplo para el mundo, en general se valora el espacio físico que se habilita, así como el despliegue de organización y logística que tiene el programa, además de los valores que promociona a la sociedad. En este sentido, Ana Cristina Nogueira en la presentación del libro Ciudadanos, calles y ciudades: Las Américas unidad por una Ciclovía (MONTEZUMA, 2011), comenta:

El concepto bogotano se ha replicado con mucha intensidad y rapidez durante los últimos años. En efecto, acondicionar las calles, avenidas, autopista durante el verano, fines de semana y días festivos, e inclusive durante la noche para el encuentro, la recreación y el deporte y el 
esparcimiento de los ciudadanos, es un evento que se ha extendido hasta Europa y se ha propagado en toda América, desde Canadá hasta Argentina, tomando diferentes denominaciones: Ciclopaseos, CicloChacao, Ciclo RecreoVía, Ciclovida, Muévete en bici, Ciclotón, Paseo Dominguero, Pasos y Pedales, Ruta RecreActiva, RecreoVía, Summer Streets, Sunday, Parways, Sunday Street, Tour del Caminante y Vía RecreActiva, entre muchos otros (p. 6).

Además de seguir el ejemplo en varias ciudades y países, la Ciclovía también ha tenido varios reconocimientos por parte de la Organización Panamericana de la Salud (OPS) y ha sido premiada por: por el Departamento Administrativo de la Función Pública (2003) al programa más reconocido y exitoso, en ese mismo año, la OMS (Organización Mundial de la Salud) y La red de Actividad Física de las Américas (RAFA/PANA) la ubica como el programa que más contribuye a que la ciudad de Bogotá, sea la más Activa y saludable de las Américas. En el Acuerdo 386 de 2009 fue declarada Patrimonio de interés social, recreativo y deportivo y en el año 2014 en el marco de la Cumbre Internacional Río+20 que se llevó a cabo en Bogotá se premió por la instaurar cultura sostenible concedido por el Foro Global de Asentamientos Urbanos y apoyado por la Programa de Naciones Unidas para el Medio Ambiente (UNEP) además recibe reconocimiento a su trayectoria de 40 años.

Se puede decir que en la primera década del siglo XXI se asiste al afianzamiento del programa Ciclovía en la ciudad de Bogotá. Aún frente a las distintas visiones políticoadministrativas distritales en esta década, en todas se reconoció la importancia del programa para la ciudad. La apuesta por fortalecer este espacio para los ciudadanos esta en reconocer la promoción que hace de una cultura urbana más comprensiva, incluyente, pacífica y humana. 
Actualmente la Ciclovía no solo tiene importancia por los modelos que propone como estilo de vida, su relevancia esta también en ser gestante del transporte alternativo en la ciudad. De lado ha quedado su articulación con el ciclismo, para sentar las bases de las ciclorrutas que despliegan "buenas prácticas" urbanas, las cuales harán sostenibles el planeta. Por lo anterior, se impulsa en distintos lugares del mundo, “[...] estableciéndose como modelos 'universales' que en algunos casos no tienen en cuenta la complejidad de los contextos locales" (MONTERO, 2016, p.1). Tal como lo señala Montero, si bien es un ejemplo para el mundo, es preciso comprender que cada contexto social es diferente y que la aplicación en las distintas ciudades del mundo requiere mucho más que un cierre de calles para permitir el tránsito de bicicletas y pedestres.

El programa ha tenido éxito porque los ciudadanos han reconocido en la Ciclovía un espacio que les permite hacer parte de su ciudad. Si bien aún falta mucho para que la recreación se asuma como un derecho ciudadano en sentidos más amplios, si es posible ver que se ha ido ganando espacios para que los bogotanos comprendan la importancia de trabajar mancomunadamente para lograr que los presupuestos del distrito asuman la responsabilidad social que les corresponde en el campo de la recreación puntualmente.

\section{Tensiones en la Apropiación del Espacio Público}

Tal como se ha venido exponiendo la recreación ha sido una mediación privilegiada para generar prácticas de apropiación de los espacios públicos de la ciudad. Este aspecto ha sido muy bien usado por las administraciones locales de turno, para mostrar 
mediáticamente los valores positivos que permiten estas prácticas en aspectos como ejercicio ciudadano activo y el derecho a la ciudad.

Escenarios tales como la Ciclovía, festivales y ferias que son promovidos por la comunidad se muestran como grandes logros y baluartes de las administraciones locales y además en la mayoría de los casos son usados como escaparate que visibiliza lo bonita e incluyente de la ciudad, generando a partir de ella, imaginarios de una urbe equitativa y plácida, que se preocupa por el bienestar general de su población, cuando en realidad enfrenta fuertes dificultades y desigualdades en el acceso a los programas recreativos y culturales.

En ese sentido, la recreación tiene una doble faceta, si bien, hoy en día la oferta en estos ámbitos ha crecido, la poca inversión de las administraciones locales termina al final por debilitar muchos programas. Por ejemplo, el programa Ciclovía ha sido subvencionado acudiendo a convenios y alianzas con empresas en el marco de la responsabilidad social empresarial y en algunos casos a recursos privados, para mantener el programa en las condiciones que se requiere. Sin embargo, ante los ciudadanos se vende como un programa sólido que cuenta con todas las garantías y respaldo de las administraciones distritales.

La recreación entendida como derecho ciudadano de optar por una vida amable, tranquila y plena con calidad, debe ser considerada como ejercicio ciudadano. De este modo, la relación espacio público-prácticas recreativas implica un compromiso de los ciudadanos con su ciudad. En este contexto, las intervenciones de los profesionales del campo de la recreación son acciones políticas que fortalecen las relaciones sociales y culturales de una comunidad. La recreación es promotora de la participación ciudadana desde acciones que no se conformen con en el carácter consultivo y que avancen a una Licere, Belo Horizonte, v.20, n.2, jun/2017 
participación deliberativa. Tal como lo expresa Yory (2011) "[...] después de todo, ciudadanía y participación van de la mano, a tal punto que no podemos entender una si no es a través de la otra” (p. 142). Por ello, La Ciclovía establece cómo en su preservación ha sido clave la defensa de los bogotanos.

Se puede decir que además de los múltiples beneficios de la recreación a la salud, el bienestar y la calidad de vida de los individuos en términos de su intervención en los espacios públicos de la ciudad se quiere resaltar en tres aspectos sus aportes: El primero, la convivencia ciudadana, segundo la inclusión social y tercero fortalecimiento a los derechos políticos:

- Convivencia ciudadana: en la medida en que los ciudadanos tengan espacios de encuentro social, es posible establecer acuerdos que permitan ejercer regulaciones consensuadas más que impuestas o normalizadas. Precisamente la importancia social y el reconocimiento del espacio público como constructor de convivencia ciudadana lo potencian como regulador social. Si bien las ciudades deben manejar códigos, normas y reglamentos, se espera que estos, tal como plantean Duhau, E. y Giglia, A. (2004), reconozcan las formas de organización propia, partiendo del hecho que los ciudadanos son quienes viven, transitan y circulan en ellas.

Los habitantes se mueven en las ciudades a partir de un conocimiento práctico y aplican normas que indican el cómo debe usarse y los comportamientos esperados en los distintos lugares. La forma en que se aprehende a vivir en la ciudad y los comportamientos públicos deseados en muchos casos se hace por imitación más que por comprensión o reflexión. En algunos casos la regulación formal perdura sin ser contextualizada con el 
momento histórico y las situaciones sociales que se tratan de regular imponen códigos disciplinarios que indican solo el qué se debe o no hacer en determinado espacio.

Estas formas de aprehender la relación con el espacio público han construido vínculos de desarraigo, deterioro del espacio, bajos niveles de sociabilidad y fomento del individualismo que conducen a la privatización, la segregación espacial y la depredación de lo público. Esto se manifiesta en la creación de controles sociales, como los sistemas de seguridad, rejas, encerramientos, policía privada, sistemas de ingreso, entre otros. En contra posición a esas formas desarraigadas de enseñar y aprehender la norma, la Ciclovía desde los años noventa se tornó en un espacio privilegiado para educar al ciudadano.

Los discursos de un espacio público, ordenado, seguro, tranquilo y plácido, han sido fundamentales para empoderar a la Ciclovía, de tal manera que exista en los usuarios una percepción de control y vigilancia garantizada por los organizadores, quien además debe enseñar a los capitalinos el cómo los acuerdos permiten el legítimo derecho a disfrutar la calle. Desde los años noventa, la pedagogía ciudadana, es clave para enseñar a vivir de manera más ordenada y tranquila en Bogotá.

- Inclusión social: la intervención de las prácticas recreativas en el espacio público genera en los participantes aceptación, respeto y reconocimiento, aspectos todos que confluyen en la inclusión social. El uso de mediaciones como el juego o acciones lúdicas permite a los individuos acceder a otros tipos de códigos de comunicación e interacción. Por ello, es fundamental que los profesionales que trabajan en este campo sean asertivos en el manejo de las técnicas que permitan a los individuos reconocer que las diferencias de pensamiento no son un impedimento para el trabajo mancomunado, que la diversidad de 
formas de ser y opiniones enriquecen el desarrollo social y que el respeto es un principio inquebrantable de las relaciones humanas.

Se puede decir que la inclusión social en un sentido básico se entiende como lo opuesto a la exclusión, es un proceso social del reconocimiento, respeto, confianza en el otro, aspectos claves en la integración a una comunidad, para Robert (2014) la inclusión social puede ser considerada como:

[...] el proceso de empoderamiento de personas y grupos, $[\ldots]$ para que participen en la sociedad y aprovechen las oportunidades. Da a la gente voz en las decisiones que influyen en su vida a fin de que puedan gozar de igual acceso a los mercados, servicios y los espacios políticos, sociales y físicos (p. 37-38).

Una recreación con vocación social contraria a una recreación pensada en pro del espectáculo y la diversión es una mediación que lleva a crear consciencia sobre los problemas que aquejan a la comunidad así como las desigualdades e inequidades urbanas en el acceso a la cultura. En tal sentido, es importante llamar a la participación ciudadana en la construcción de una política urbana equitativa, con la comprensión que el espacio público requiere de una mayor atención donde la recreación y la cultura son aspectos fundamentales en el desarrollo humano de los habitantes.

En el crecimiento y la organización del espacio público de la ciudad, la recreación se ha manifestado como una necesidad general, los espacios de socialización, la preocupación por el tiempo libre de los ciudadanos y otras formas de relación permiten hacer de estos espacios físicos y simbólicos facilitadores de inclusión y reconocimiento social.

\section{- Fortalece los derechos políticos de los ciudadanos}


Cuando se conquista un espacio físico para el disfrute ciudadano, se está velando por un derecho fundamental como lo es la recreación. Todos los seres humanos merecen espacios físicos accesibles, espacios para su encuentro y pleno desarrollo de la ciudadanía, aspectos que son posibles gracias a que existe un espacio público que permita una vida colectiva. En la medida en que la población de las ciudades crece descontroladamente es necesario concientizar a los ciudadanos sobre la necesidad de un espacio público suficiente y en buenas condiciones.

Para nadie es un secreto que el crecimiento acelerado de las ciudades ha comprometido el espacio público para sus habitantes, según el último reporte técnico de la Defensoría del Espacio Público de la ciudad de Bogotá (2016) muestra que:

El indicador de compacidad corregida nos muestra la relación que se está generando en la ciudad del espacio público frente a las dinámicas de la construcción, donde se generan zonas densamente construidas con pocos espacios abiertos para la recreación y el deporte (s.p.).

Tal como lo evidencia este estudio sobre el espacio público de la ciudad de Bogotá, cada vez más se vulneran las condiciones para disfrutar la ciudad, se ha privilegiada la edificación sobre la calidad de vida de los habitantes. Por tanto, es urgente que la ciudadanía defienda, proteja y preserve tanto los espacios naturales como los espacios físicos de todos y para todos, además de luchar por garantizar los derechos a recrearse, expresarse libremente y socializar con otros, derrotando modelos en donde el Estado endilga a los ciudadanos su responsabilidad a asumir la recreación y por tanto estimula modelos de inequidad y desigualdad social:

La crisis económica estructural de los equipamientos culturales, es producto de la crisis general del Estado nacional y del advenimiento del proyecto neoliberal en Latinoamérica, que llevó al Estado a abandonar la 
garantía y tutela de los derechos sociales y culturales convirtiéndolos en bienes bajo la lógica del mercado (TABARES y MOLINA, 2013, p. 39).

Para el año 2013 las Encuestas Bienales de Cultura $(\mathrm{EBC})^{8}$ realizadas por la Secretaria de Cultura Recreación y Deporte en cabeza del Observatorio de Cultura reconocían que el 47,5\% de las personas residentes en Bogotá es completamente necesario recrearse, aspecto que permite denotar la importancia de ésta en la vida cotidiana de los ciudadanos. Sin embargo, en el año 2011 se reportaba que el 72, 61\% de los bogotanos reconocían que la principal actividad con fines recreativos era visitar centros comerciales, seguido de reunirse con los amigos o familiares en un $71,48 \%$ y en tercer lugar se encuentran los paseos por la ciudad con un $65,40 \%$. Según el análisis realizado por el Observatorio de Culturas (2012), ir a un centro comercial punteada como la actividad recreativa más realizada por cuatro factores fundamentales: la amplia oferta concentrada en estos lugares (cine, comidas, compras, espectáculos y juegos infantiles, entre otros); en segundo lugar a la poca exigencia de planificación para asistir a este sitio (oferta dispuesta en cualquier momento); tercero, una supuesta gratuidad para acceder al espacio, lo que permite que la familia participe y cuarto la sensación de seguridad que este espacio controlado ofrece a sus visitantes.

De este análisis quisiera resaltarse por un lado, el incremento que tiene un tipo de recreación ligada al consumo y el espectáculo y por otro, el acceso libre, disponible y

\footnotetext{
${ }^{8}$ No se toma el último registro de la Encuesta Bienal del 2015 porque el ítem recreación desaparece. La palabra recreación queda asociada al uso de los parques y equipamientos. En el 2011 se evaluaba el ítem Actividad física, deporte y recreación, haciéndose explicita la pregunta por las actividades recreativas más frecuentes. En la encuesta del año 2013 se abre un ítem denominado Recreación y entretenimiento, este acogía preguntas por el espacio público, la calle, los grafitis en la ciudad. En la última Encuesta Bienal (2015) el ítem recreación y esparcimiento es reemplazado por espacio público.
} 
seguro con el que se asocia a los centros comerciales, en contravía de la calle como escenario de peligro y riesgo para los ciudadanos y especialmente para los niños y mujeres. Precisamente la encuesta del 2013 pregunta por el significado de la calle además de ser un espacio de transito, dando cuenta cómo para el $86,9 \%$ de las personas es considerada un espacio de peligro, seguido de un $84,6 \%$ que la consideran un espacio de encuentro y con un $78 \%$ espacio de entretenimiento.

Se resalta como los datos que consideran la calle como espacio de peligro y espacio de encuentro son muy cercanos, es posible decir, que para muchos bogotanos es importante este espacio como eje socializador, lo cual indica un reto para los profesionales de la recreación, quienes pueden encargarse de modificar el imaginario de peligro que tienen muchos ciudadanos por hacer de la calle un espacio para todos. Se persiste en decir que cuando los espacios públicos se ocupan con actividades de recreación y deporte, el miedo y la inseguridad son desplazados, las personas se sienten seguras y la calle, los parques y las plazas retoman su sentido, el encuentro social.

Por tanto, se hace una invitación para interpelar la lógica del mercado que propende por una sociedad más inequitativa, con ofertas culturales excluyentes donde fortalece un imaginario de la ciudad de los miedos que a su vez genera una amenaza para fortalecer el espacio público como eje que fortalece los derechos políticos y civiles. Contrario a este proyecto, se presenta la perspectiva que invierte y defiende la calle, el parque y la plaza para el disfrute, el encuentro y la cohesión social. Es urgente estudiar y generar indicadores que permitan mostrar el impacto de la recreación en el espacio público de las ciudades con mediciones que demuestren el fortalecimiento de la convivencia ciudadanía, 
la inclusión, el reconocimiento social y la defensa de los derechos políticos de los ciudadanos.

\section{A Modo de Conclusión}

Sí bien son conocidos los aportes en los ámbitos sociales, culturales y de salud que ofrece la recreación a las comunidades en el mejoramiento de su calidad de vida, aún sigue siendo complejo justificar ante las administraciones de la ciudad el sostenimiento para llevar a cabo los programas en esta área.

La recreación puede genera dos modelos de relación con el espacio público: uno para agenciar de manera pasiva posturas de ciudadanía por la vía del adiestramiento, donde la participación ciudadana queda regulada y relegada al cumplimiento de normas en el buen uso del espacio público, y el otro, para generar un ejercicio ciudadano deliberativo y propositivo que construya apropiación, respeto y preservación del espacio a partir de vivencias y experiencias recreativas de gozo, satisfacción personal que contribuyan a la construcción de cohesión y compromiso ciudadano.

\section{REFERENCIAS}

ANÓNIMO. Circuito ciclistico de la tranquilidad. Periódico EI Tiempo, n. 14, dec, 1974.

BORJA, J. Ciudadanía y espacio público. Revista Foro, n. 40, p. 67-80, 2000. . Prólogo. En: En: SÁNCHEZ, D. \& DOMÍNGUEZ, L. (Coord.) Identidad y

Espacio Público. Barcelona: Editorial Gedisa S, A., 2014

CULTURA RECREACIÓN Y DEPORTE. Observatorio de Cultura. Encuestas Bienales de Cultura $(2011,2013,2015)$. Recuperadas de: 
http://www.culturarecreacionydeporte.gov.co/es/observatorio-de-culturas/encuesta-bienalde-culturas.

DEFENSORÍA DEL ESPACIO PÚBLICO Primer reporte técnico de indicadores de espacio público, $2016 . \quad$ Recuperado de http://observatorio.dadep.gov.co/sites/default/files/primerreporteindicadores.pdf .

DUHAU, E. \& GIGLIA A. Conflicto por el espacio y el orden urbano. Revista estudios Demográficos urbanos, v. 19, n. 2 (56), p. 257-288, 2004.

ESCOBAR, A. La invención del tercer mundo. Bogotá: Norma, 1996.

GÓMEZ. M. Ciclovías, deporte al aire libre y mucho más. Periódico El Tiempo. Recuperación digital, 1984. Acesso em: 25 ago. 2014.

INSTITUTO DISTRITAL DE RECREACIÓN Y DEPORTE - IDRH. Ciclovía: laboratorio para el futuro. Colombia, Bogotá: IDRD, 2007.

LEFEBVRE, H. (1974/2013). La producción del espacio. Madrid: Capitán Swing.

LINDÓN, A. El habitar la ciudad, las redes topológicas del urbanita y la figura del transeúnte. En: En: SÁNCHEZ, D. \& DOMÍNGUEZ, L. (Coord.) Identidad y Espacio Público. Barcelona: Editorial Gedisa S, A., 2014.

MONTEZUMA R. Ciudadanos, calles y ciudades: las Américas unidas por una Ciclovía. Colombia, Bogotá: Organización Panamericana de la Salud, Centro de transporte sustentable México, Universidad del Rosario, Fundación movilidad, Red de Ciudades por la Bicicleta, Fundación ciudad humana, 2011.

MONTERO, S. Worlding Bogotá's Ciclovía From Urban Experiment to International "Best Practice”. Latin America Perspectives, v. 20, n. 30, p. 1-2), 2016.

NULLVALUE. La Ciclovía hoy está de fiesta. Periódico EI Tiempo, 26 sep. 1999.

OBSERVATORIO DE CULTURAS. Boletín n. 6. Recreación, el reino de la libertad. Observaciones de ciudad. Alcaldía Mayor de Bogotá: Bogotá, 2012.

PARDO, M. Territorialidades Cívicas: Espacio público y cultura urbana en Bogotá. Bogotá: Universidad Externado de Colombia, 2008.

PÉRGOLIS, J. Bogotá fragmentada: cultura, espacio urbano finales del siglo XX. Bogotá: Tercer Mundo Editores, Universidad Piloto de Colombia, 1998.

PALACIOS, R. Mitin en favor de la cicla. Periódico EI Tiempo, 16 dec. 1974.

PÉREZ, M. Ciclo-solución a la recreación. Periódico El Tiempo. Recuperación digital, 23 feb. 1992 . 
RAMÍREZ, O. Ciclovías: La ciudad para el ciudadano. En: (s.a.) Ciclovías Bogotá para el ciudadano. Bogotá: Editolaser, 1983.

ROBERT, M. Desigualdad e inclusión social en las Américas: elementos clave, tendencias recientes y caminos hacia el futuro. En: Desigualdad e Inclusión Social en las Américas. Washington, D.C. OEA, 2014.

TABARES, F. \& MOLINA V. Desigualdades socio-espaciales en la distribución de los equipamientos culturales de América Latina. Bogotá: Fonámbulos, 2013.

SALDARRIAGA, A. ¿Qué tan público es el espacio público? Magazín Dominical Periódico El Espectador, 03 en. 1999.

SENNETT. R. EI declive del hombre público. Barcelona: Anagrama, 2011.

SILVA, A. Imaginarios Urbanos. Bogotá: Arango Editores, 2006.

UNIVERSIDAD NACIONAL DE COLOMBIA. Línea base y evaluación de satisfacción recreación y deporte. Informe final no publicado (8 de Marzo de 2004), 2003.

Estudio de Satisfacción de las actividades realizadas por el IDRD. Tomo (I). Ciclovía-Recreovía. Centro de Investigaciones para el Desarrollo. Facultad de Ciencias Económicas. Diciembre, 2009.

VALERA. S. (2014). La identidad social urbana como instrumento para mejorar el bienestar humano. En: Sánchez, D. \& Domínguez, L. (Coord.) Identidad y Espacio Público. Barcelona: Editorial Gedisa S, A.

YORY, C. M. Espacio público y derecho a la ciudad. En: Espacio público y derecho a la ciudad. Bogotá: Universidad Javeriana, 2011.

ZAMBRANO, F. Observatorio de Cultura Urbana. Construcción del espacio público Tres parques de Bogotá: Nacional, Simón Bolívar, El Tunal. Bogotá: Centro de investigaciones de la Universidad de los Andes y la Alcaldía de Bogotá: Bogotá, 2003.

\section{Endereço da Autora:}

Astrid Bibiana Rodríguez Cortés

Calle 64 a n. 57-23 torre 2 apt. 1202

Bogotá - Colômbia

Endereço Eletrônico: abrodriguez@pedagogica.edu.co 